\title{
PENTALOGÍA DE CANTRELL Y BANDAS AMNIÓTICAS: REPORTE DE UN CASO Y REVISIÓN DE LA LITERATURA
}

\section{Pentalogy of Cantrell and amniotic bands: A case report and review of the literature}

\author{
Wilmar Saldarriaga-Gil, MD, MSc ${ }^{1}$; Sebastián Ayala-Zapata ${ }^{2}$; \\ Julián Andrés Ramírez-Cheyne, $\mathrm{MD}^{3}$; Carolina Isaza, $\mathrm{MD}, \mathrm{MSc}^{4}$
}

Recibido: diciembre 4/13 - Aceptado: agosto 15/14

\section{RESUMEN}

Objetivo: reportar un caso de pentalogía de Cantrell asociado a acranea, hendidura facial medial y reducción de las extremidades derechas originado por bandas amnióticas. Realizar una revisión de la literatura sobre la patogénesis de las bandas amnióticas y los hallazgos en el caso reportado.

Materiales y métodos: se presenta el caso de un recién nacido muerto, con malformaciones congénitas antes mencionadas, hijo de madre primigestante de 15 años con 34 semanas de gestación, que consultó al Hospital Universitario del Valle (HUV), hospital de nivel III y IV de referencia en obstetricia, de la red pública del suroccidente colombiano. Como fuente de información se utilizó la historia clínica, fotografías del recién nacido y los Rayos X.

1 Especialista en Ginecología y Obstetricia. Embriología-Genética. Profesor Asociado, Departamentos de Ginecología y Obstetricia y Morfología, Hospital Universitario del Valle. Director del Programa Medicina y Cirugía, Universidad del Valle, Cali (Colombia). wsaldarriaga0608@yahoo.com

2 Estudiante, Tercer año, Pregrado de Medicina y Cirugía, Universidad del Valle, Cali (Colombia).

3 Candidato a Magíster en Ciencias Básicas Médicas. Embriología y Genética, Universidad del Valle. Grupo de Malformaciones Congénitas Perinatales y Dismorfología (MACOS), Universidad del Valle. Profesor Asistente, Departamento Morfología, Facultad de Salud, Universidad del Valle, Cali (Colombia)

4 Magíster en Ciencias Básicas Médicas, Énfasis Embriología y Genética, Grupo de Malformaciones Congénitas Perinatales y Dismorfología (MACOS), Universidad del Valle. Profesora Titular, Departamento de Morfología, Facultad de Salud, Universidad del Valle, Cali (Colombia).
Se realizó una búsqueda de literatura con las palabras clave "Pentalogy of Cantrell and amniotic band syndrome", "Limb body wall complex and amniotic band syndrome" en las bases de datos Medline vía PubMed en inglés y español, sin límite de tiempo; se buscaron reportes de caso, revisiones de tema y series de casos.

Resultados: se encontraron treinta artículos, se analizaron todas las referencias y se halló que catorce corresponden a reportes de caso, ocho a revisiones de tema y ocho a series de casos.

Conclusiones: se reporta un caso de pentalogía de Cantrell, posiblemente por bandas amnióticas originadas en el desarrollo embrionario. El caso se clasificó como complejo cuerpo-extremidad (LBWC), secundario a bandas amnióticas.

Palabras clave: pentalogía de Cantrell, síndrome de bandas amnióticas, complejo cuerpo-extremidad.

\section{ABSTRACT}

Objective: To report a Pentalogy of Cantrell case associated with acrania, medial facial cleft and reduction of the right limbs as a result of amniotic bands; and to conduct a review of the literature on the pathogenesis of amniotic bands and the findings of the reported case.

Materials and methods: Case report of a demised neonate with the above mentionated 
congenital malformations born to a 15 year-old primigravida in her 34 weeks of gestation who came to Hospital Universitario del Valle (HUV), a level III and IV referral hospital for obstetrics belonging to the public network in south-western Colombia. The clinical record, photographs of the neonate and X-Ray images were used as the sources of information. A search in the literature was conducted using the terms "Pentalogy of Cantrell and amniotic band syndrome", "Limb body wall complex and amniotic band syndrome" through PubMed in the Medline databases in English and Spanish, with no time limitation; the search included case reports, topic reviews and case series. Results: Overall, 30 articles were found and all the references were analysed; 14 were case reports, 8 were topic reviews and 8 were case series.

Conclusions: Report of a Pentalogy of Cantrell case, probably resulting from amniotic bands arising during embryo development. It was classified as a limb body wall complex (LBWC), secondary to amniotic bands.

Key words: Pentalogy of Cantrell, amniotic band syndrome, limb body complex.

\section{INTRODUCCIÓN}

La pentalogía de Cantrell (PC) es una rara anomalía congénita con una prevalencia entre 1 en 65.000 a 1 en 200.000 nacimientos (1), en la que ocurre un defecto primario en el epigastrio que compromete la porción inferior del tórax y la superior del abdomen, implicando a su vez los órganos adyacentes. Cantrell informó, en 1958, cinco casos con esta anomalía (2).

La pentalogía de Cantrell se caracteriza por cinco hallazgos: defecto de la pared toracoabdominal, hipoplasia de la porción inferior del esternón, hernia diafragmática anterior, pericardio pobremente formado y anomalías cardiacas, entre ellas la más representativa es la ectopia cordis (2, 3). La etiología de la PC es heterogénea, en la mayoría de los casos no se encuentra una causa específica; sin embargo, se han reportado familias con patrón de herencia ligado al cromosoma X, casos con trisomías cromosómicas, y en otros se ha documentado exposición a teratógenos (4-6). La asociación etiológica entre PC y el síndrome de bandas amnióticas es rara, y pocos casos se han reportado en la literatura $(7,8)$.

El síndrome de bandas amnióticas (SBA) tiene un rango de prevalencia de 1 en 11.200 a 1 en 18.000 recién nacidos vivos $(9,10)$, y se ha encontrado relacionado a madres primigestantes, jóvenes, con bajo nivel educativo, ocurrencia de fiebre y sangrados en el primer trimestre de gestación, exposición a medicamentos, tabaco y diabetes (11, 12). El SBA consiste en un conjunto de anomalías congénitas secundarias al efecto destructivo de una banda de tejido amniótico que se adhiere al embrión o feto en desarrollo, que puede producir anillos de constricción o amputaciones usualmente distales en extremidades (13-16). Con menor frecuencia se ha asociado a defectos de la pared toraco-abdominal, exencefalia/acranea, con o sin hendidura facial, y anormalidades de las extremidades; esta asociación se conoce como complejo cuerpo-extremidad, síndrome del tallo corporal o LBWC (del inglés limb body wall complex) (17-19).

El objetivo de este estudio es reportar un caso de pentalogía de Cantrell asociado a acranea, hendidura facial medial y reducción de las extremidades derechas, originado por bandas amnióticas. Asimismo, se realiza una revisión de la literatura sobre la patogénesis de las bandas amnióticas y los hallazgos en el caso reportado.

\section{PRESENTACIÓN DEL CASO}

Recién nacido muerto, de sexo masculino, hijo de unión no consanguínea de madre primigestante de 15 años, de estrato socioeconómico bajo, quien realizó un pobre control prenatal iniciado a las 20 semanas de gestación, con ecografías a las 26 y 32 semanas que mostraron anencefalia, onfalocele y pie equinovaro. A las 34 semanas consulta al Hospital Universitario de Valle en Cali (Colombia), hospital de III y IV nivel de referencia en obstetricia, de 
la red pública del suroccidente colombiano, que atiende alrededor de 5.000 nacimientos por año de mujeres de estratos socioeconómicos 1, 2 y, en menor proporción, del estrato 3, del régimen subsidiado por el Estado, con patologías materno-fetales de alta complejidad.

En la atención a la gestante se encontró un feto muerto y se decidió la finalización del embarazo. Se atiende parto vaginal obteniéndose un mortinato de $1405 \mathrm{~g}$, con percentil menor de 3 para la edad gestacional. El examen físico y los estudios radiográficos evidenciaron defecto de pared toraco-abdominal que incluía el esternón, a través del cual protruían el corazón (ectopia cordis) y el intestino; hendidura facial medial, exencefalia/ anencefalia; acortamiento en miembro superior derecho, con hueso radio displásico; reducción del miembro inferior derecho caracterizado por mesomelia con acortamiento de la tibia y fíbula; pie equinovaro, pseudosindactilia con amputación de artejos del pie derecho y anillos de constricción en el segundo y tercer dedos del pie, que sugieren que las malformaciones son secundarias al efecto mecánico de bandas amnióticas (figuras 1 y 2). Se tomó muestra de sangre para cariotipo por punción del cordón umbilical y no se obtuvo crecimiento en el cultivo de linfocitos.

Se encontró como exposición importante que la madre trabajó durante el periodo periconcepcional en una mina de oro informal donde se usaba mercurio para separar las partículas del metal.

Aspectos éticos. El reporte del caso y la publicación de las fotografías se hacen con la firma del consentimiento informado de la madre. Se protegió la confidencialidad de las identidades del feto y la paciente.

\section{MATERIALES Y MÉTODOS}

A partir de las preguntas sobre la patogénesis de las bandas amnióticas y los hallazgos del caso reportado se realizó una búsqueda bibliográfica en la base de datos Medline vía PubMed, utilizando como encabezados de búsqueda: "Pentalogy of Cantrell and amniotic band syndrome" y "Limb body wall complex and amniotic band syndrome"; se buscaron artículos en inglés y en español, revisiones de tema, reportes de caso y series de casos, sin límite de tiempo.

\section{RESULTADOS}

Se encontraron treinta artículos; su análisis arrojó que catorce corresponden a reportes de caso, ocho a revisiones de tema y ocho a series de casos.

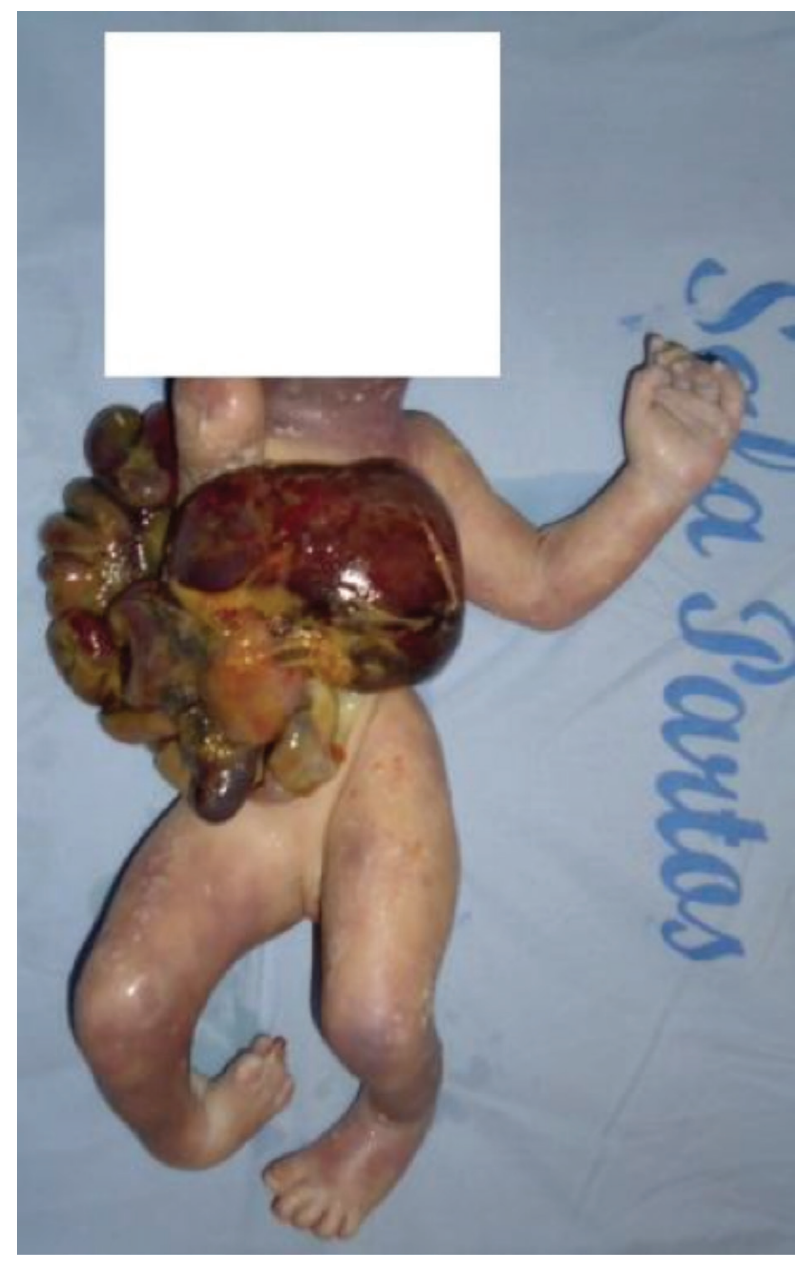

Figura 1. Vista anterior del recién nacido con múltiples anomalías congénitas. Obsérvese defecto de pared toracoabdominal (más del lado derecho a nivel abdominal) a través del cual protruyen el corazón y el intestino, acortamiento en miembro superior derecho, pie equinovaro derecho, pseudosindactilia con amputación en artejos del pie derecho, y anillos de constricción en el segundo y tercer dedo del pie derecho. 


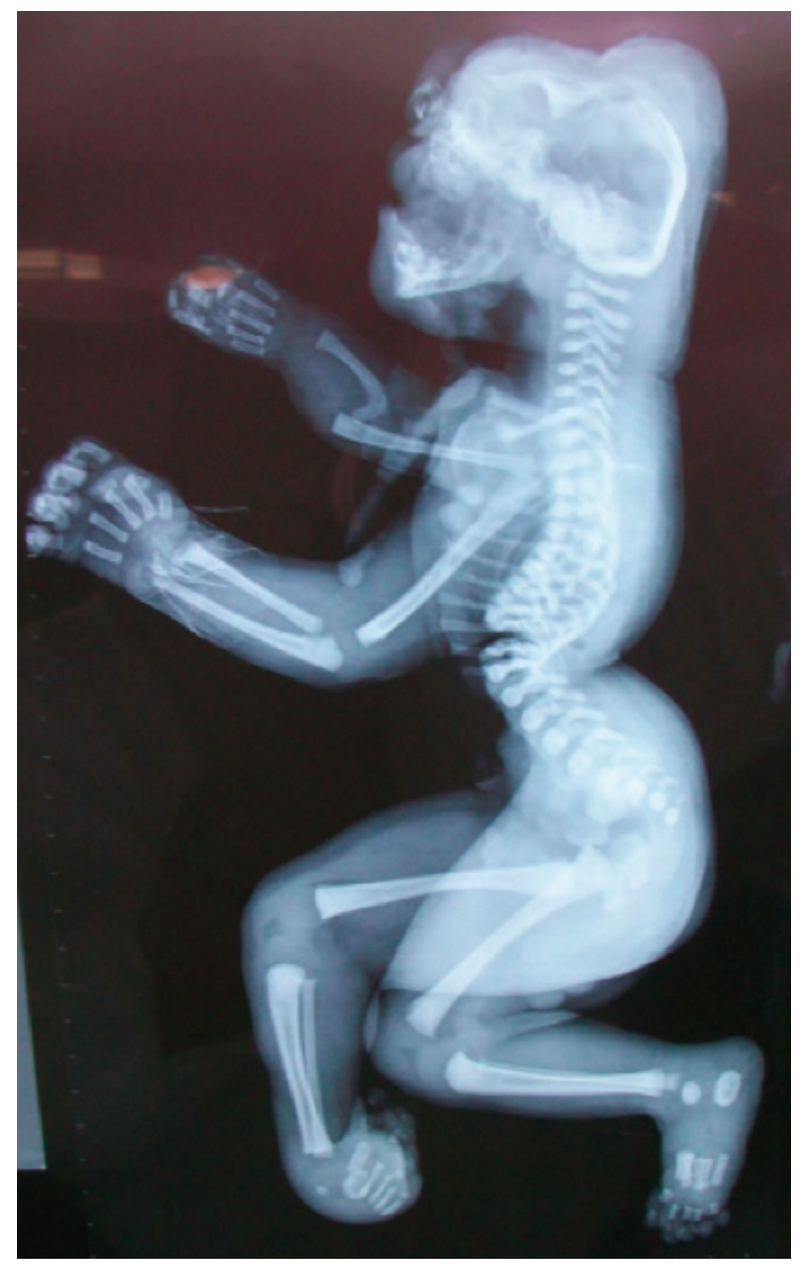

Figura 2. Obsérvese agenesia de ulna derecha, radio derecho displásico, acortamiento de tibia y fíbula derecha, ausencia de metatarsianos del pie derecho, alteraciones de las curvaturas del plano sagital de la columna vertebral.

Patogénesis. Existen diferentes teorías etiológicas para explicar la fisiopatogenia del síndrome de bandas amnióticas. Torpin, en 1965, postuló que la ruptura prematura del amnios permitiría el paso de líquido amniótico a la interfase amniocoriónica, quedando el embrión expuesto a la superficie interna de la cavidad coriónica, estimulando la formación de bandas amnióticas fibrosas que pueden adherirse al embrión y producir daños consecuentes (20-23). Si las bandas amnióticas se producen antes del día 45 posterior a la fecundación, pueden interferir con el desarrollo de estructuras y órganos en formación, interrumpiendo la embriogénesis, afectando los plegamientos craneal caudal y transversal, produciéndose defectos en la pared toraco-abdominal, en el cierre del tubo neural, en la formación de la cara con la fusión de los 5 primordios faciales y el crecimiento, y segmentación de las yemas de las extremidades $(24,25)$. Esta asociación se conoce en la literatura como complejo cuerpo-extremidad, síndrome del tallo corporal o, en inglés, limb body wall complex (LBWC) $(17,26,27)$.

En el caso aquí presentado se propone que la banda amniótica afectó dramáticamente al embrión desde la cuarta semana del desarrollo, en la etapa denominada blastogénesis $(28,29)$, produciendo el defecto de la pared toraco-abdominal, la ectopia cordis y la evisceración de otros órganos; la exencefalia/acranea, la fisura facial y la reducción de las extremidades. Cakiroglu et al., en 2014, reportaron la asociación entre PC y agenesia de miembro superior izquierdo en un embarazo gemelar, los autores no lo asociaron a bandas amnióticas (30). Chen et al., en 2008, reportaron la asociación entre PC y exencefalia, artrogriposis, defecto del pulgar izquierdo y acortamiento de la extremidad superior, sin asociarlo a bandas amnióticas (31). Schüppler et al., en 1994, reportaron un caso de PC con gran defecto del cráneo y encefalocele, y lo asociaron a síndrome de bandas amnióticas (7). En 1993, Peer et al. reportaron también un caso de PC con exencefalia y síndrome de bandas amnióticas (8).

Otra teoría sobre la fisiopatología del SBA la propuso Streeter quien postuló que primero ocurriría una anomalía primaria fetal con alteración del disco germinal, generando defectos como fallas en el cierre del tubo neural y de la pared toraco- abdominal, que producirían una respuesta inflamatoria del amnios contiguo, y de manera secundaria se desarrollaría una banda fibrosa (21, 32, 33). Esta teoría también podría explicar los hallazgos del caso aquí reportado, postulando que la acranea, el defecto toraco-abdominal y la evisceración consecuente produjeron las bandas amnióticas que afectaron las extremidades. 
Posteriormente se propuso la "teoría vascular", que sugiere que el proceso se inicia con una necrosis vascular del amnios que produce disrupciones que predisponen a adherencia de partes embrionarias o fetales al amnios en los sitios necróticos y así se generan las bandas adheridas $(34,35)$.

Una vez se produce la banda amniótica esta se adhiere al embrión o feto en desarrollo, lo que puede producir constricción sobre estructuras ya formadas y llevar a una disminución del aporte sanguíneo a los tejidos implicados, dejando cicatrices en forma de anillos en partes distales de dedos o regiones proximales como brazo y muslo, o medias como antebrazo o pierna, pudiéndose en ocasiones producir amputación de esas estructuras $(13,14)$. El caso aquí reportado presentó hallazgos similares en el miembro inferior derecho.

Diagnóstico diferencial. El caso aquí reportado, además de los hallazgos clásicos de PC, presentó acranea y fisura facial medial, lo que hace proponer como diagnóstico diferencial el síndrome toraco-abdominal (THAS, del inglés thoraco abdominal syndrome), que se caracteriza por la asociación inconstante entre defectos de la pared toracoabdominal y otros defectos de línea media como acranea y hendidura facial medial, y por tener un origen genético con un patrón de herencia dominante ligado al cromosoma $\mathrm{X}$, reportándose marcadores genéticos en la región Xq25-q26 (36). Dado que en el caso reportado no se encontraron antecedentes familiares de casos similares -lo que hace poco probable un origen genético- y que los hallazgos en el miembro inferior derecho eran concluyentes de la presencia de bandas amnióticas, se excluyó este diagnóstico.

Asociaciones. La madre del caso aquí reseñado presentó varias asociaciones reportadas en el SBA (ser adolescente, primigestante y tener bajo nivel educativo); además, estar expuesta a mercurio en el periodo periconcepcional; esta exposición se relaciona con bajo peso al nacer en modelos animales (37), en humanos se ha informado asociación con otras anomalías congénitas como alteración neurológica conocida como la enfermedad de Minamata $(38,39)$ y no con el SBA.

\section{CONCLUSIÓN}

Se reporta un caso de pentalogía de Cantrell asociado a acranea, hendidura facial medial y reducción de las extremidades derechas, anomalías causadas por bandas amnióticas originadas en la cuarta semana del desarrollo embrionario, pudiendo clasificar el caso como Complejo Cuerpo-Extremidad, LBWC. Este es el primer caso reportado con asociación de PC, exencefalia/acranea, hendidura facial y reducción de extremidades originados por bandas amnióticas.

\section{REFERENCIAS}

1. Jafarian AH, Omidi AA, Fazel A, Sadeghian H, Joushan B. Pentalogy of Cantrell: a case report. J Res Med Sci. 2011;16:105-9.

2. Cantrell JR, Haller JA, Ravitch MM. A syndrome of congenital defects involving the abdominal wall, sternum, diaphragm, pericardium and heart. Surg Gynecol Obstet. 1958;107:602.

3. Engum SA. Embryology, sternal clefts, ectopiacordis, and Cantrell's pentalogy. Seminars in Pediatric Surgery. 2008;17:154-60.

4. Chen CP. Syndromes and disorders associated with omphalocele (II): OEIS complex of Cantrell. Taiwan J Obstet Gynecol. 2007;46:103-10.

5. Pachajoa H. Pentalogía de Cantrell en un recién nacido expuesto en útero a misoprostol. Rev Chil Obstet Ginecol. 2010;75:47-9.

6. Soria J, Guzmán A, Hernández I. Presentación y discusión de un paciente con pentalogía de Cantrell. Rev Cubana Obstetricia Ginecología. 2004;30: 34-9.

7. Schüppler U, Weisner D, Schollmeyer T, Grillo M, Franz W. Combination of Cantrell pentalogy and amniotic band syndrome: a case report. Zentralbl Gynakol. 1994;116:115-9.

8. Peer D, Moroder W, Delucca A. Prenatal diagnosis of the pentalogy of Cantrell combined with exencephaly and amniotic band syndrome. Ultraschall Med. 1993;14:94-5.

9. Orioli IM, Ribeiro MG, Castilla EE. Clinical and epidemiological studies of amniotic deformity, 
adhesion and mutilation sequence in a South American population. Am J Med Genet A. 2003;118:135-45.

10. Martínez-Frias ML. Epidemiological characteristics of amniotic band sequence (ABS) and body wall complex (BWC): are they two different entities? Am J Med Genet. 1997;73:176-9.

11. Cignini P, Giorlandino C, Padula F, Dugo N, Cafà EV, Spata AJ. Epidemiology and risk factors of amniotic band syndrome, or ADAM sequence. Spata AJ Prenat Med. 2012;6:59-63.

12. Werler MM, Louik C, Mitchell AA. Epidemiologic analysis of maternal factors and amniotic band defects. Birth Defects Res A Clin Mol Teratol. 2003;67:68-72.

13. Pedersen TK, Thomsen SG. Spontaneous resolution of amniotic bands. Ultrasound Obstet Gynecol. 2001;18:673-4.

14. Rivas R, Juárez A, Islas L, Durán MA, Oviedo I. Síndrome de bandas amnióticas asociado a secuencia Potter. Un caso de autopsia. Rev Mex Pediatr. 2005;72:78-81.

15. Poeuf B, Samson P, Magalón G. Amniotic band syndrome. Chir Main. 2008;27:S136-47.

16. Martínez Ml, Bermejo E, Rodríguez E. Body stalk defects, body wall defects, amniotic bands with and without body wall defects, and gastroschisis: comparative epidemiology. Am J Med Genet. 20001;92:13-8.

17. Levy R, Lacombe D, Rougier Y, Camus E. Limb body wall complex and amniotic band sequence in sibs. Am J Med Genet Part A. 2007;143A:2682-7.

18. Russo R, Vecchione R. Limb body wall complex: craniofacial defects as a distinctive factor. Birth Defects Orig Artic Ser. 1996;30:157-64.

19. Chen CP, Tzen CY, Chang TY, Yeh LF, Wang W. Prenatal diagnosis of acrania associated with facial defects, amniotic bands and limb-body wall complex. Ultrasound Obstet Gynecol. 2002;20:94-5.

20. Torpin R. Amniochorionicmesoblastic fibrous strings and amniotic bands: associated constricting fetal malformations or fetal death. Am J Obstet Gynecol. 1965;91:65-75.

21. Moerman P, Fryns JP, Vandenberghe K, Lauweryns JM. Constrictive amniotic bands, amniotic adhesions, and limb body wall complex: discrete disruption sequences with pathogenic overlap. Am J Med Genet. 1992;42: 470-9.
22. Halder A. Amniotic band syndrome and/or limb body wall complex: split or lump. Appl Clin Genet. 2010;3:7-15.

23. Yang SS. ADAM sequence and innocent amniotic band: manifestations of early amnion rupture. Am J Med Genet. 1990;37:562-8.

24. Saldarriaga W, Isaza C. Presentación de 4 casos de defectos de blastogénesis. Rev Med Colombia. 2005;36:18-25.

25. Da Silva G, Cammarata-Scalisi F, González-Coira M, Lacruz MA, Rendon B. Síndrome de bridas amnióticas, a propósito de 3 casos clínicos. Rev Chil Pediatr. 2008;79:172-80.

26. van Allen MI, Curry C, Gallagher L. Limb body wall complex: I. Pathogenesis. Am J Med Genet. 1987;28:529-48.

27. Das SK, Maharana SS, Subudhi M, Rao PV. The limbabdominal wall complex defects, a form of amniotic band syndrome: a rare case report. J Clin Diagn Res. 2013;7:543-4.

28. Opitz JM, Zanni G, Reynolds JF Jr, GilbertBarness E. Defects of blastogenesis. Am J Med Genet. 2002;115:269-86.

29. Hartwig NG, Vermeij-Keers C, De Vries HE, Kagie M, Kragt H. Limb body wall malformation complex: an embryologic etiology? Hum Pathol. 1989;20: 1071-7.

30. Cakiroglu Y, Doger E, Yildirim Kopuk S, Babaoglu K, Caliskan E, Yucesoy G. prenatal diagnosis of cantrell's pentalogy associated with agenesis of left limb in a twin pregnancy. Case Report. Obstet Gynecol. 2014;2014:3142-84.

31. Chen CP, Tzen CY, Chen CY, Tsai FJ, Wang W. Concomitant exencephaly and limb defects associated with pentalogy of Cantrell. Taiwan J Obstet Gynecol. 2008;47:476-7.

32. Bamforth JS. Amniotic band sequence: Streeter's hypothesis reexamined. Am J Med Genet 1992;44: 280-7.

33. Speert H. Memorable medical mentors IX George L Streeter (1873-1948). Obstet Gynecol Surv. 2005; 60:3-6.

34. van Allen M, Curry C, Walden C, Gallagher L, Patten R. Limb-body wall complex: II. Limb and spine defects. Am J Med Genet. 1987;28:549-65. 
35. Hunter AG, Seaver LH, Stevenson RE. Limbbody wall defect. Is there a defensible hypothesis and can it explain all the associated anomalies? Am J Med Genet A. 2011;155A:2045-59.

36. Mckusick V, Bocchini C, Kniffin C. Online Mendelian Inheritance in Man OMIM [base de datos en Internet]. Thoracoabdominal Syndrome: THAS. Baltimore: Johns Hopkins University. [Visitado 2013 nov. 20]. Disponible en: http://www.ncbi.nlm.nih.gov/omim
37. Gandhi DN, Panchal GM, Dhull DK. Influence of gestational exposure on the effects of prenatal exposure to methyl mercury on postnatal development in rats. Cent Eur J Public Health. 2013;21:30-5.

38. Kessler R. The Minamata Convention on Mercury: a first step toward protecting future generations. Environ Health Perspect. 2013;121:A304-9.

39. Karada M. Congenital Minamata disease: intrauterine methylmercury poisoning. Teratology. 1978;18:285-8.

\section{Conflicto de intereses: ninguno declarado.}

